Voix et Images

voixetimages

\title{
Autour de quelques morts
}

\section{Michel Biron}

Volume 24, numéro 2 (71), hiver 1999

Poésie québécoise et histoire littéraire

URI : https://id.erudit.org/iderudit/201436ar

DOI : https://doi.org/10.7202/201436ar

Aller au sommaire du numéro

\section{Éditeur(s)}

Université du Québec à Montréal

\section{ISSN}

0318-9201 (imprimé)

1705-933X (numérique)

Découvrir la revue

\section{Citer cet article}

Biron, M. (1999). Autour de quelques morts. Voix et Images, 24(2), 407-412.

https://doi.org/10.7202/201436ar d'utilisation que vous pouvez consulter en ligne.

https://apropos.erudit.org/fr/usagers/politique-dutilisation/ 


\title{
Roman
}

\section{Autour de quelques morts}

\author{
Michel Biron, Université du Québec à Montréal
}

Après L'acquittement ${ }^{1}$, qui lui a valu l'an dernier le Grand prix du livre de Montréal, Gaétan Soucy publie une sorte de conte romanesque intitulé La petite fille qui aimait trop les allumettes ${ }^{2}$. À michemin entre les facéties de Jacques Ferron ${ }^{3}$ et la philosophie de Ludwig Wittgenstein (cité en exergue des deux textes), les romans de Soucy lancent toutes sortes d'étincelles à gauche et à droite, cherchant parfois leur style dans une gymnastique syntaxique qui tient de l'exercice davantage que de l'audace, mais trouvant aussi des bonheurs d'écriture qui expliquent en partie l'engouement de la critique montréalaise. La petite fille qui aimait trop les allumettes combine un style en apparence extrêmement simple, plein de formules orales, d'onomatopées puériles et de familiarités phatiques, et une écriture coupante comme du fil de fer barbelé, qui déchiquette les moindres mollesses sentimentales. L'enfance n'existe ici qu'à travers l'expérience de la mort, mais d'une mort qui s'intériorise à travers l'expérience de détachement que constitue l'écriture. Les mots sont des "poupées de cendre" (p. 174), dit la narratrice, cette "petite fille qui aimait trop les allumettes" et qui joue avec les mots comme avec le feu.

Le roman s'ouvre sur la découverte du père, pendu dans sa chambre. C'est ainsi que le narrateur (qui deviendra en cours de route une narratrice) et son frère prennent soudainement, bien malgré eux, l'univers en main. Mais comment exister, comment vivre dans un monde qui n'est plus soumis aux ordres du père? Les deux enfants s'étaient tellement habitués aux règles paternelles, ils avaient tellement bien intégré le bon usage des "horions", qu'ils se retrouvent figés, frappés de stupeur. Devant eux s'ouvre un monde parfaitement inconnu, le monde du dehors. Toute leur existence s'est jusque-là déroulée à l'intérieur du domaine paternel, sans contact ou presque avec le village qui se trouve de l'autre côté de la pinède. Isolés, mais instruits (le père fortuné possédait une bibliothèque remplie de dictionnaires, de romans de chevalerie et des ouvrages contenant de grandes vérités comme l'éthique de spinoza, les mémoires du duc de saint-simon - les minuscules sont dans le texte - et bien sûr la bible), ils se disent prêts à faire "face à la musique" (p. 15).

Et quelle musique! Imaginez un requiem profane joué au fond d'une forêt devant deux enfants qui enterrent le corps de leur père suicidé en menant un sabbat de tous les diables. Ajoutez que la narratrice et son frère idiot ont une sœurette à moitié vivante enveloppée comme une momie dans le fond du caveau familial, juste à côté du squelette de 
la mère. Elle s'appelle Juste Châtiment et ressemble à un personnage de Beckett, incapable de mourir tout à fait. La narratrice, prénommée Alice comme l'héroïne du conte, n'a jamais connu personne en dehors de cet enfer incestueux. Le jour où elle se rend, seule, au village, en quête d'un cercueil pour son père, elle $y$ rencontre des gens étranges qui finissent par la reconnaître et par enquêter chez elle. Au plus fort de la scène d'apocalypse qui s'ensuit, le frère écervelé tire à coups de carabine du haut de sa forteresse et tue un inspecteur des mines pendant que brûle la bibliothèque et qu'Alice s'échappe, avec pour seul bagage le bébé qu'elle porte dans son ventre, ayant décidé au préalable qu'elle immolerait son grimoire comme on sacrifiait jadis des animaux. Un requiem, disions-nous? Plutôt une musique à réveiller les morts, très peu recommandable pour le recueillement et le repos.

Il est difficile de donner une idée précise de l'agitation extrême qui s'empare de l'écriture sous l'effet de cette musique endiablée, qui veut nous entraîner à l'assaut du vide. L'effet de surexcitation ne fonctionne pleinement que si le lecteur accepte de lire à une certaine vitesse et s'il dévore le texte d'un bout à l'autre, sans trop s'attarder à ses scories et à ses procédés. Voịci un exemple de cet affolement narratif associé à la cruauté humaine et qui passe par la syntaxe même du récit. Le frère vient d'enduire de térébenthine des perdrix et il les caresse à présent avec la flamme d'une allumette:

Les perdrix, que voulez-vous, elles s'affolaient, c'est humain. Elles sont parties s'assommer au plus sacrant dans les carreaux de la chapelle, à la queue leu leu, pour achever le supplice et les énervements de se voir en tel appareil de feu, et j'aurais fait de même, garanti. (p. 43)

Ce qui frappe dans ce court passage, c'est moins la cruauté du geste du frère et la violence de la mort en elle-même, qui sont neutralisées et réduites à un pur spectacle, poétique de surcroît ("appareil de feu"), que la forme même du texte, tout entier tourné vers le lecteur - vers un certain lecteur, celui qui partage les mêmes tics de langage et qui peut ainsi participer à la "production du sens". La mort n'est mise à distance qu'au prix du rapprochement immédiat entre le texte et un lecteur qui connaît et pratique le mélange de babil et de poésie dont raffole la narratrice. La rhétorique de ce passage vise en effet à produire un effet d'identification avec un lecteur d'ici et d'aujourd'hui (la deuxième phrase est du charabia pour un lecteur d'ailleurs ou d'une autre époque). Tel est le sens de la formule phatique "que voulez-vous", de la compassion ironique de la narratrice ("c'est humain "), de l'intrusion abrupte du "je" qui n'ajoute strictement rien sinon justement la nécessité de s'identifier à ces victimes (de même que le lecteur s'identifie à la narratrice-victime), enfin et surtout de l'énoncé "au plus sacrant" et du mot final "garanti" qui relèvent tous deux du registre familier. Les mots glissent d'un registre à l'autre et suggèrent que le langage n'est authentique et personnel que s'il emballe le lecteur, appelé à faire contrepoids à une réalité pétrifiée, mortifère. Comme la narratrice devant le spectacle insupportable des perdrix en feu se jetant sur les vitres 
de l'église, nous devons dire, nous aussi, devant cette écriture enflammée: “j'aurais fait de même, garanti ".

Assez curieusement, ce changement de registre ne se produit pas seulement à l'échelle de la phrase. D'un roman à l'autre, Soucy change également de registre, ou plus justement de style, comme s'il n'avait pas vraiment trouvé le sien. Dans L'acquittement, le héros Louis Bapaume faisait face à une autre musique (il incarnait un musicien incapable de réussir l'oratorio qu'il avait rêvé de composer), même s'il affirmait similairement que "rien n'est réel ". Les mots n'étaient pas pour lui des "poupées de cendre", mais des "prismes" à travers lesquels il percevait les choses et les êtres. Il y avait, dans cette image du prisme, toute une poétique du regard: devant un monde ni transparent ni opaque, le sujet voit double, triple, comme s'il multipliait les points de réfraction et mêlait malicieusement l'espace du sens, de la sensation et de la sensibilité.

D'où vient que la force de cette prose cruelle, sèche et chaleureuse à la fois me touche beaucoup moins dans le dernier roman? Le livre refermé, le lecteur est essoufflé. $L a$ petite fille qui aimait trop les allumettes est un roman intelligent, fulgurant, mais, une fois leur course terminée, les personnages meurent aussitôt, êtres de papier incapables de se transporter au-delà du grimoire dont ils sont issus. L'art du portrait, si vif et si précis dans L'acquittement, cède la place à une exaspérante allégorie de l'inceste et surtout à un repli de la littérature sur elle-même. Que la littérature renvoie à la littérature n'a pourtant rien pour effrayer ni surprendre le lecteur contemporain, mais il y a une passion pour la littérature qui a quelque chose de suspect et de mécanique, du moment qu'elle sert à racheter le manque de réalité des choses. Trop ostentatoire, l'écriture de La petite fille est inégale, incapable de garder le ton juste et de suivre le rythme épuisant de cette valse à mille temps. Autour du mort, l'enfant s'émerveille de sa propre candeur, de sa propre écriture. Il se voit en train de danser avec les oiseaux, avec les mots-oiseaux :
Je m'étonne toujours de constater qu'une fois la première rafale passée, je suis capable d'une telle indifférence à ce qui peut m'arriver de terrible ici-bas, c'est dans mon caractère, comme. Je tourne lentement sur moi-même avec ma jupe amie de saturne qui est ma planète, et je ris sans que ça paraisse dans le petit autel de mon silence, pareille comme elle. Mes pieds vont légers à l'exemple des oiseaux qu font leur vol autour de mon corps et qui ont la couleur de mes yeux, car tous les oiseaux valsent avec moi, c'est mon secret, même ceux qui sont à l'autre bout de la terre. (p. 89)

Rien n'est plus difficile sans doute que de feindre la désinvolture, de représenter rigoureusement cette fausse candeur de l'enfant devant les mots et la réalité, comme dans cette courte phrase qui allie un infinitif très littéraire et un mot d'enfant: "Et monsieur de se mettre à pleurer, peuh" (p. 30). Après l'étonnement initial, que voulez-vous, le lecteur voit assez vite les rouages de cette écriture trop prévisible, comme.

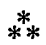

Du style à l'écriture, enseignait jadis Roland Barthes ${ }^{5}$, il y a toute la 
distance qui sépare la parole brute, biologique, d'un discours défini par sa destination, donc par sa nature sociale. Est-ce un hasard si j'ai le sentiment, à lire de front plusieurs romans québécois d'aujourd'hui, que, sous des styles on ne peut plus variables, ils se soutiennent d'une même écriture, fondée sur le plaisir du détachement, sur la "désentimentalisation" du roman, mais aussi sur un certain besoin d'adoration (tantôt la littérature, tantôt la pureté de l'enfance)? Voici le début du Désarroi du matelot, deuxième roman du poète Michael Delisle:

\section{À proprement parler, j'ai fait sauter Jean-François Vézina-Côté, quatorze ans. Décapité par la déflagration. Sa tête rousse a volé dans les airs et s'est nichée dans l'immense sapin bleu sur le parterre de ses parents ${ }^{6}$. (p. 11)}

Beauté et banalité du crime, évoqué à travers une expression figée ("À proprement parler") qui signale d'entrée de jeu au lecteur que le récit commènce à froid, loin du drame. Là encore, comme chez Soucy, le récit frappe par son "impertinence", au sens à la fois moral et linguistique du terme: le ton est déplacé par rapport à la gravité de la situation. On ne badine pas avec la mort, à moins qu'on ne soit un spécialiste, un criminel endurci, ce que n'est certainement pas le matelot de Delisle. Criminel malgré lui, il se trouve, comme le dit le titre, en plein désarroi et n'a d'autre lieu pour se confesser que la mission de Sister Russell, au centre-ville de Montréal. Elle y recueille des êtres sans feu ni lieu et leur donne l'occasion de s'exprimer en toute impunité. Il doit parler et dire ce que Sister appelle "the cruel truth" (p. 13). Mais vous pourriez lui ouvrir le corps et le torturer de n'importe quelle façon, il semble avoir perdu l'usage de la parole, du moins cet usage selon lequel la parole sert à exprimer des émotions et à maintenir le lien social. Il préfère porter une photographie de sa victime, de son cher ange, "[s]on fer rouge, [s]on signe" (p. 24), comme si le seul langage qu'il comprenait était celui de l'image.

Ce talisman joue dans le roman un rôle majeur: non seulement le héros conserve-t-il ainsi l'image de l'enfant tué sur sa poitrine, mais il se projette également tout entier dans cette figure de l'innocence, tentant de se l'approprier et d'entretenir avec elle un dialogue latéral, loin du monde, loin de sa propre jeunesse (durant laquelle il fut matelot, d'où le titre), loin de toute communication ordinaire. Exemple:

\begin{abstract}
Assis sur le marchepied, je regarde la photo de mon ange. Il est tout usé, les couleurs se sont affadies et l'encre s'est déposée contre le plastique. Je caresse ses cheveux roux avec mon pouce. Tu affiches un sourire de convention, convenu comme ta vie avant ta décollation. Chérubin, que tu saches ta chance d'évoluer dans la pureté infatigable. Ici, la lumière tombe et les oiseaux s'inquiètent; on appelle ça le soir. Te souviens-tu du soir, JeanFrançois Vézina-Côté? (p. 61)
\end{abstract}

Il ne s'agit même pas de culpabilité à vrai dire, car les crimes (le matelot en a commis d'autres dont il minimise l'importance) n'ont pas de poids en eux-mêmes. Cela tient plutôt de l'hommage funèbre (à la "pureté infatigable"). On se souvient qu'à Montréal, il y a quelques années, un jeune garçon est 
mort tragiquement à la suite d'une explosion provoquée par quelque groupe lié au crime organisé. Le désarroi du matelot semble écrit à partir de là : nous assistons à la rencontre (attendue) du meurtrier et de sa victime par la voie de la fiction.

Il y a dans ce roman la matière d'une nouvelle, tout au plus. On se demande un peu ce que vient faire la deuxième partie, dans laquelle un nouveau personnage assez insignifiant entre en scène. Il s'agit de monsieur Harrisson, père de famille qui profite d'un héritage pour quitter son emploi d'agent de surveillance et pour se lancer, à son propre compte cette fois, sur la piste de Richard Daudelin, le narrateur de la première partie. Grâce à son enquête et au changement de point de vue narratif, le lecteur suit le héros pas à pas et le voit agir de l'extérieur. Mais il perd du coup le fil du roman, qui se termine, de façon assez glauque, dans une chambre de motel, par le meurtre hautement programmé du détective sortant de sa poche une autre photo de l'enfant assassiné. L'idolâtrie a ses lois, surtout lorsqu'il s'agit d'une forme d'adoration toute personnelle, incommunicable, comme celle du matelot pour sa victime.

\section{$* *$}

Après avoir donné la parole à une morte dans L'ingratitude ${ }^{7}$, Ying Chen la donne à une jeune femme qui réincarne une chanteuse d'opéra, jadis épouse d'un prince polygame. Au lieu de s'effacer de sa mémoire, cette vie antérieure lui remonte à la gorge alors qu'elle vit en pleine modernité. Une modernité trom- peuse, symbolisée par son mari qui est professeur d'archéologie et qui s'y connaît donc un peu en matière d'ancêtres. Mais cette connaissance tout intellectuelle du passé ne lui permet pas de comprendre sa femme, car celle-ci connaît le passé d'un point de vue tout différent, organique plutôt que cérébral. Lui, c'est un moderne, un scientifique, un homme de terrain et un homme de son temps: il coïncide parfaitement avec son époque. Les "absences" ou les folies de sa femme, qu'il adore par ailleurs (comme on adore ce qui tient du mystère le plus pur), lui paraissent les signes d'une faiblesse de l'esprit. Au départ, les allusions à une vie antérieure lui semblent même assez sympathiques, originales, féminines pour tout dire. Mais au fur et à mesure que cette anamnèse se développe, l'amusement du mari se transforme en agacement puis en rejet. L'immobilité de la narratrice ne constitue pas seulement une sorte de résistance passive au tumulte moderne: elle en constitue la négation pure et simple.

Le mari a beau tout essayer pour ramener sa femme à la raison, à la raison moderne s'entend, c'est peine perdue. La mémoire corporelle de la narratrice se développe comme un cancer, une maladie dévastatrice. Le pitoyable désarroi du mari n'y peut rien: sa femme habite un autre monde que le sien. Elle n'appartient peut-être même pas à l'univers romanesque proprement dit. Sa folie ne relève pas de la passion et n'a rien à voir avec les désirs insatisfaits de Madame Bovary: elle relève de l'allégorie. L'autre vie qu'elle ne parvient pas à oublier n'a pourtant rien de très enviable: elle ne fut qu'une épouse dans un harem 
dont le prince préférait la conversation de ses serviteurs à celle de ses femmes. Elle eut toutefois un amant, un esclave justement, dont elle causa la perte (il fut décapité). C'est cet amant, bien sûr, qui lui revient en mémoire, c'est-à-dire l'amour dominateur et meurtrier qu'elle éprouva jadis pour un homme qui lui était entièrement soumis. Mais, pas plus qu'elle n'est Emma Bovary, la narratrice ne ressemble à quelque Phèdre par qui le malheur serait arrivé. L'autre vie ne tient pas lieu de modèle: elle permet de fuir la tyrannie du présent, cette "modernité joviale ${ }^{8}$ " (p. 62) comme l'appelle le mari avec son habituel sens critique. Elle permet aussi de fuir la tyrannie de la filiation, de rompre la chaîne du temps et d'inventer non des modèles, mais des ancêtres. Pré- et post-moderne, l'héroïne devient à soi-même son propre ancêtre, crée son propre personnage, fabrique ses propres morts, ceux qui habitent sa mémoire et définissent son identité. De là aussi son sentiment de précéder ses parents ("J'ai vécu avant mes parents" (p. 9), découvre-t-elle avec une fierté mélancolique), de les avoir engendrés elle-même. L'effort de déconditionnement auquel elle consent presque involontairement l'oblige à naître de plus loin et introduit l'archaïsme au cœeur même de la modernité.

Curieuse fable que cet Immobile, à la fois plus ambitieuse que les romans précédents de Ying Chen et plus artificielle. À travers le jeu un peu forcé de la réincarnation, cette fable porte un jugement sur la modernité. L'idée d'écrire un roman contre la vie moderne a quelque chose de séduisant, mais cela reste une idée, si originale soit-elle (ce qui ne va pas de soi). Difficile de l'oublier, difficile de ne pas la sentir toujours à l'œuvre, prenant à certains moments la place de l'œuvre. En plusieurs endroits, le besoin d'explication est tel qu'il écrase la fable et contraint l'imagination. Les dés sont pipés dès le début: le triste mari ne peut pas arracher sa femme à son immobilité ontologique et leur relation, faussée d'entrée de jeu, semble extrêmement superficielle. Comme Charles Bovary, comme Thésée aussi, il appartient au monde ordinaire, immédiat; il ne comprend pas que sa femme ne puisse pas se défaire de son fardeau, qu'elle ne sache ni fuir ni suivre les autres. Il finira d'ailleurs par retourner à son travail, résigné à perdre son amour, sans trop comprendre pourquoi (tous les traitements, de la psychiatrie au Nouvel Âge, n'y peuvent rien). Elle reste là, immobile, devant un monde qui s'éloigne d'elle ou qui la foule sans la remarquer, comme une pierre sur le chemin. Elle attend, venue d'on ne sait où, morte et vivante à la fois, jetant au hasard des vents la poussière de sa mémoire au visage ennuyé des contemporains.

1. Gaétan Soucy, L'acquittement, Montréal, Boréal, 1997, 123 p.

2. Gaétan Soucy, La petite fille qui aimait trop les allumettes, Montréal, Boréal, 1998, $180 \mathrm{p}$.

3. L'hérö̈ne a quelque chose de la Tinamer de Portanqueu de L'amélanchier (Montréal, Typo, 1992, 207 p.).

4. Gaétan Soucy, L'acquittement, op. cit., p. 122 .

5. Roland Barthes, Le degré zéro de l'écriture suivi de Nouveaux essais critiques, Paris, Seuil, coll. "Points", 1972, p. 11-17.

6. Michael Delisle, Le désarroi du matelot, Montréal, Leméac, 1998, 139 p.

7. Ying Chen, L'ingratitude, Montréal/Arles, Leméac/Actes Sud, 1995, 133 p.

8. Ying Chen, Immóbile, Montréal, Boréal, 1998, $156 \mathrm{p}$. 